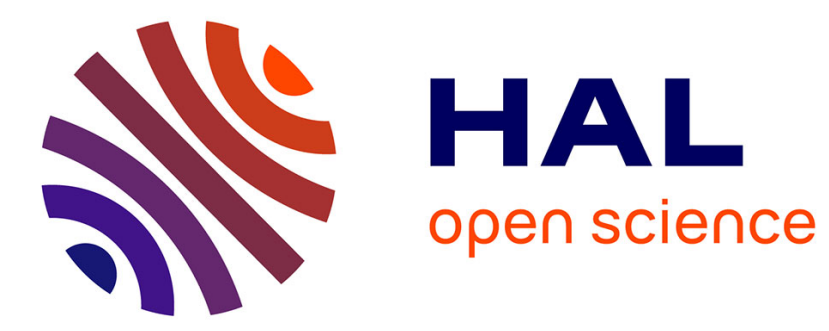

\title{
Inelastic x-ray scattering from valence electrons near absorption edges of FeTe and TiSe2
}

Bernardo Barbiellini, J. N. Hancock, C. Monney, Yves Joly, G. Ghiringhelli, L. Braicovich, T. Schmitt

\section{- To cite this version:}

Bernardo Barbiellini, J. N. Hancock, C. Monney, Yves Joly, G. Ghiringhelli, et al.. Inelastic x-ray scattering from valence electrons near absorption edges of FeTe and TiSe2. Physical Review B: Condensed Matter and Materials Physics (1998-2015), 2014, 89 (23), pp.235138. 10.1103/PhysRevB.89.235138 . hal-01016739

\section{HAL Id: hal-01016739 \\ https://hal.science/hal-01016739}

Submitted on 1 Jul 2014

HAL is a multi-disciplinary open access archive for the deposit and dissemination of scientific research documents, whether they are published or not. The documents may come from teaching and research institutions in France or abroad, or from public or private research centers.
L'archive ouverte pluridisciplinaire HAL, est destinée au dépôt et à la diffusion de documents scientifiques de niveau recherche, publiés ou non, émanant des établissements d'enseignement et de recherche français ou étrangers, des laboratoires publics ou privés. 


\title{
Inelastic x-ray scattering from valence electrons near absorption edges of $\mathrm{FeTe}^{\mathrm{T}}$ and $\mathrm{TiSe}_{2}$
}

\author{
B. Barbiellini, ${ }^{1}$ J. N. Hancock, ${ }^{2,3}$ C. Monney, ${ }^{4}$ Y. Joly,,${ }^{5,6}$ G. Ghiringhelli, ${ }^{7}$ L. Braicovich, ${ }^{7}$ and T. Schmitt ${ }^{8}$ \\ ${ }^{1}$ Physics Department, Northeastern University, Boston, Massachusetts 02115, USA \\ ${ }^{2}$ Department of Physics, University of Connecticut, Storrs, Connecticut 06269, USA \\ ${ }^{3}$ Institute for Materials Science, 97 North Eagleville Road, Storrs, Connecticut 06269, USA \\ ${ }^{4}$ Fritz-Haber-Insitut der Max-Planck-Gesellschaft, Faradayweg 4-6, D-14195 Berlin, Germany \\ ${ }^{5}$ Université Grenoble Alpes, Institut NEEL, F-38000 Grenoble, France \\ ${ }^{6}$ CNRS, Institut NEEL, F-38042 Grenoble, France \\ ${ }^{7}$ CNR/SPIN and Dipartimento di Fisica, Politecnico di Milano, Piazza Leonardo da Vinci 32, I-20133 Milano, Italy \\ ${ }^{8}$ Paul Scherrer Institut, CH-5232 Villigen PSI, Switzerland
}

(Received 23 April 2014; revised manuscript received 10 June 2014; published 30 June 2014)

\begin{abstract}
We study resonant inelastic x-ray scattering (RIXS) peaks corresponding to low-energy particle-hole excited states of metallic FeTe and semimetallic TiSe $\mathrm{T}_{2}$ for photon incident energy tuned near the $L_{3}$ absorption edge of $\mathrm{Fe}$ and Ti, respectively. We show that the cross-section amplitudes are well described within a renormalization-group theory where the effect of the core electrons is captured by effective dielectric functions expressed in terms of the the atomic scattering parameters $f_{1}$ of $\mathrm{Fe}$ and Ti. This method can be used to extract the dynamical structure factor from experimental RIXS spectra in metallic systems.
\end{abstract}

DOI: 10.1103/PhysRevB.89.235138

PACS number(s): 78.70.Ck, 71.15.Qe, 71.15.Mb

\section{INTRODUCTION}

Inelastic x-ray scattering (IXS) probes charge and spin electronic excitations in condensed matter, but the corresponding cross section is generally small [1], especially the magnetic IXS cross section [2]. By tuning the incident energy near an x-ray absorption edge a cross-section enhancement can be observed [3-10]. Work by Kramers, Heisenberg, Weiskopf, and Wigner provides the foundation for quantitative approaches [11] for resonant IXS (RIXS). Nevertheless, some small disagreements between theory and experiment recently observed both in liquids [12] and in solids [13,14] suggest that the Kramers-Heisenberg $(\mathrm{KH})$ theory misses some inelastic processes, especially at incident energy tuned slightly lower than the absorption threshold.

The KH expression based on nonrelativistic second-order perturbation theory for the interaction between light and matter describes well the radiation reemitted by atoms after illumination by primary radiation (e.g., resonant $\mathrm{x}$-ray emission) but cannot fully describe collision processes between weakly bound electrons and light quanta [15]. The scattering of an $\mathrm{x}$-ray photon from nearly free electrons involves both energy and momentum conservation of an almost isolated system formed by two particles, namely, the electron and the photon. The Thirring theorem states that the cross section of this Compton-like effect with all radiative corrections reduces in the nonrelativistic limit to the Thomson cross section (i.e., nonrelativistic limit of the Klein-Nishima cross section [16]). The only effect of the vacuum or of the medium is to renormalize the Thomson cross section [17].

In order to explore the scattering of nearly free electrons by a photon tuned near the binding energy of a core level, we have introduced a scheme based on the renormalization-group (RG) theory [18] able to cast the complicated RIXS problem to a much simpler IXS problem in an effective medium described by an effective dielectric function $\epsilon$, which integrates out the effect of the core electrons. In the RG approach, a differential equation describing the evolution of the coupling constant with screening yields the renormalization factor $\eta$ for the effective Thomson cross section in the polarizable medium. This method effectively allows one to calculate the incident photon energy dependence of the RIXS cross section in the case of a metallic system. Recently, Haverkort [19] has shown that effective models based on the absorption spectra can give a good description of RIXS, although his approach was applied to spin excitations in strongly correlated systems rather than charge excitation in metallic systems as in the present case.

The present work studies high-resolution measurements [20,21] near the $\mathrm{Fe} L_{3}$ edge of $\mathrm{Fe}_{1.087} \mathrm{Te}$ and the Ti $L_{3}$ edge of $\mathrm{TiSe}_{2}$ by considering low-energy particle-hole scattering peaks as a function of incident photon energy. Previous RIXS studies at the $L_{3}$ edge of $\mathrm{Cu}$ and $\mathrm{Ni}$ performed at lower resolution have neglected momentum conservation $[22,23]$. Based on the changes in intensity of these peaks, we show that the resonant scattering cross section can be described via a renormalization factor $\eta$ multiplying the nonresonant IXS cross section. In principle, the knowledge of the dynamical structure factor permits us to extract band structure information from measured dispersing electron-hole excitations. It also allows reconstructions of the density propagator of a system [24], which yields the time-dependent linear response of the system to a point perturbation. Therefore, momentum-dependent RIXS can become a unique window for visualizing the dynamics of weakly bound electrons in condensed matter.

An outline of this paper is as follows. Section II summarizes our model. In Sec. III, we present the methods for the electronic structure calculations and for the RIXS experiments. The results of the calculations are presented and compared with experimental results in Sec. IV, and the conclusions are given in Sec. V.

\section{MODEL}

In our model, the low-energy-loss scattering leads to particle-hole excited states of the valence electron system described by the double-differential scattering cross section, 
which is the product of the effective Thomson scattering cross section $d \sigma_{T h}^{*} / d \Omega$ and of the dynamical structure factor $S(\mathbf{q}, \omega)$ :

$$
\frac{d^{2} \sigma}{d \Omega d \omega}=\frac{d \sigma_{T h}^{*}}{d \Omega} S(\mathbf{q}, \omega) .
$$

For particle-hole excitations the dynamical structure factor is given by [25]

$$
S(\mathbf{q}, \omega)=\int_{-\omega}^{0} \frac{d E}{2 \pi} \int \frac{d^{3} p}{(2 \pi)^{3}} A(\mathbf{p}, E) A(\mathbf{p}+\mathbf{q}, E+\omega),
$$

where $(\mathbf{q}, \omega)$ are the momentum and energy transfer $A(\mathbf{p}, E)=$ $(1 / \pi) \operatorname{Im}[G(\mathbf{p}, E)]$ is the imaginary part of the electron Green's function $G(\mathbf{p}, E)$. The spectral function $A(\mathbf{p}, E)$ can also be expressed in terms of the Dyson orbitals $g_{v}$ as follows:

$$
A(\mathbf{p}, \omega)=\sum_{\nu}\left|g_{v}(\mathbf{p})\right|^{2} A_{\nu}(\omega)
$$

and

$$
A_{v}(\omega)=\frac{\gamma}{\pi\left[\left(\omega-\epsilon_{v}\right)^{2}+\gamma^{2}\right]},
$$

where $\epsilon_{v}$ is the excitation energy of the Dyson orbital $g_{v}(\mathbf{r})$ and $\gamma$ is an inverse lifetime. In the present case, the Dyson orbitals are given by Bloch wave functions, where $v=(\mathbf{k}, n)$ is given by the Bloch wave vector $\mathbf{k}$ and by the energy band index $n$. Therefore, one can write

$$
g_{\mathbf{k}, n}(\mathbf{r})=\exp (i \mathbf{k} \cdot \mathbf{r}) \sum_{\mathbf{G}} C_{\mathbf{G}}^{\mathbf{k}, n} \exp (-i \mathbf{G} \cdot \mathbf{r})
$$

whose momentum density is

$$
\left|g_{\mathbf{k}, n}(\mathbf{p})\right|^{2}=\sum_{\mathbf{G}} \delta(\mathbf{p}-\mathbf{k}+\mathbf{G})\left|C_{\mathbf{G}}^{\mathbf{k}, n}\right|^{2} .
$$

The Fourier coefficients $C_{\mathbf{G}}^{\mathbf{k}, n}$ of the periodic part of $g_{\mathbf{k}, n}(\mathbf{r})$ are labeled by the reciprocal vectors $\mathbf{G}$. Therefore, the spectral functions enforce the energy conservation near the Fermi level and the momentum conservation in the first Brillouin zone.

Because of the proximity of the $L_{3}$ absorption threshold, the incoming photon generates a set of virtual intermediate states involving a $2 p$ core hole and a corresponding electron excited in a $3 d$ state which can be described by an effective dielectric function $\epsilon$ experienced by the valence electrons. When the scattering process is completed, it leaves behind low-lying valence excited states conserving energy and crystal momentum, and the Thomson cross section $\sigma_{T h}$ of this resonant scattering is increased by the factor $\eta$ compared to the nonresonant cross section. The solution of the renormalizationgroup equation gives [18]

$$
\eta=\exp \left[\frac{2}{3 \alpha}\left(\epsilon / \epsilon_{0}-1\right)\right]
$$

where $\alpha$ is the fine-structure constant, $\epsilon$ is the real part of an effective dielectric function for the valence electrons as a function of energy $\omega$, and $\epsilon_{0}$ is the dielectric constant in vacuum. One can connect $\epsilon$ to the atomic scattering parameter $f_{1}$ through the formula

$$
\frac{\epsilon}{\epsilon_{0}}=1-\left(\frac{\omega_{P}}{\omega}\right)^{2} f_{1},
$$

where $\omega_{P}$ is the plasma frequency for the valence electron gas at the Fermi level, which can be written as

$$
\omega_{P}=47.1 r_{s}^{-3 / 2} \mathrm{eV},
$$

where $r_{s}$ is the radius containing a valence electron. The parameter $f_{1}$ is related to the absorption coefficient $\mu(E)$ via the Kramers-Krönig transform [26],

$$
f_{1}(E)=Z+\frac{2}{\pi} \int_{0}^{\infty} \frac{\omega_{P}^{2} \mu(\omega)}{E^{2}-\omega^{2}} d \omega,
$$

where $Z$ is the atomic charge. The cross-section enhancement occurs only if $f_{1}$ is negative. The parameter $f_{1}$ can be also determined by reflectivity experiments [27,28]. The enhancement term is therefore given by

$$
\eta=\exp \left[-\frac{2}{3 \alpha}\left(\frac{\omega_{P}}{\omega}\right)^{2} f_{1}\right],
$$

and it can be expanded in a power series of the parameter $f_{1}$ which contains a Lorentz oscillator [16] for the core $2 p$ electrons appearing also in the KH treatment of RIXS.

\section{COMPUTATIONAL AND EXPERIMENTAL METHODS}

We have calculated $f_{1}$ from first principles using the program FDMNES [29] within the time-dependent density functional theory (TDDFT) [30]. First, we performed localdensity-approximation self-consistent calculations for $\mathrm{FeTe}$ and $\mathrm{TiSe}_{2}$, and then we computed $f_{1}$ at the Fe $L_{3}$ edge and at the $\mathrm{Ti} L_{3}$ edge using TDDFT. The calculations for $\mathrm{Ti}$ are performed for the $L_{3}$ and the $L_{2}$ edges since the $L_{2}$ edge influences the $f_{1}$ value near the $L_{3}$ edge. The influence of the $L_{2}$ edge can be neglected in Fe since the separation between the $L_{2}$ and the $L_{3}$ edges is larger in this case. The crystal structure of FeTe is tetragonal with point group $P 4 / \mathrm{nmm}$ and lattice constants $a=b=3.8215 \AA, c=6.2695 \AA$, while the crystal structure of $\mathrm{TiSe}_{2}$ has a point group $P-3 m 1$ with lattice constants $a=b=3.54 \AA$ and $c=6.008 \AA$.

The experimental RIXS spectra considered in this paper were taken at the ADRESS beam line [31] of the Swiss Light Source, Paul Scherrer Institut, using the SAXES spectrometer [32]. A scattering angle of $130^{\circ}$ was used, and the samples were measured at an incidence angle of $65^{\circ}$, using $\sigma$-polarized light. At the $\mathrm{Ti} L_{3}$ edge, the combined energy resolution was 90 meV. TiSe ${ }_{2}$ samples were measured at $16 \mathrm{~K}$. At the Fe $L_{3}$ edge, the combined energy resolution was $73 \mathrm{meV}$. The FeTe samples were measured near $20 \mathrm{~K}$. Our results showing the inelastic peak height dependence on the incident photon energy were obtained by integrating the RIXS intensity between 0.1 and $4.0 \mathrm{eV}$ energy loss for FeTe and between 0.1 and $0.6 \mathrm{eV}$ energy loss for $\mathrm{TiSe}_{2}$, after having subtracted a linear background and the elastic line. Our RIXS spectra were also corrected for self-absorption effects, following the method used in Ref. [13].

\section{RESULTS}

We show in Fig. 1 a resonant feature below the Fe $L_{3}$ threshold of $\mathrm{Fe}_{1.087} \mathrm{Te}$. The subthreshold peak energy locks to a constant value of $0.9 \mathrm{eV}$ while the RIXS line shape preserves its shape. Interestingly, the momentum transfer of this peak is $q=0.51 \AA$, and the energy transfer of about 


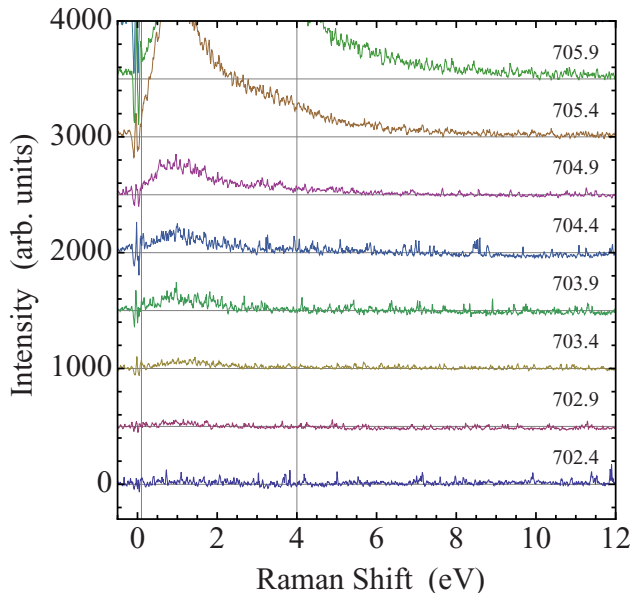

FIG. 1. (Color online) Focus on the RIXS subthreshold feature of $\mathrm{Fe}_{1.087} \mathrm{Te}$. We concentrate on the $0.9-\mathrm{eV}$ energy loss; however, we consider the area below 0.1 and $4.0 \mathrm{eV}$ to estimate the enhancement of the peak. This spectrum has been corrected for self-absorption effects, following the method used in Ref. [13]. The elastic line has also been removed.

$0.9 \mathrm{eV}$ corresponds to the recoil energy of an electron or a hole $q^{2} /\left(2 m^{*}\right)$ with an effective mass $m^{*} \sim 1$. This feature is also consistent with excitations associated with bands crossing the Fermi level around the $\Gamma$ point forming small hole pockets and around the $M$ point giving small electron pockets [33]. Similar Fermi surfaces are also found in the $\mathrm{LaO}_{1-x} \mathrm{~F}_{x} \mathrm{FeAs}$ iron pnictide compounds $[34,35]$.

The calculated $f_{1}$ as a function of incident energy is shown in the top frame of Fig. 2 together with $f_{1}$ data from total electron yield experiments in pure iron [36]. The agreement

\section{$\mathrm{Fe}_{3}$ edge of $\mathrm{FeTe}$}
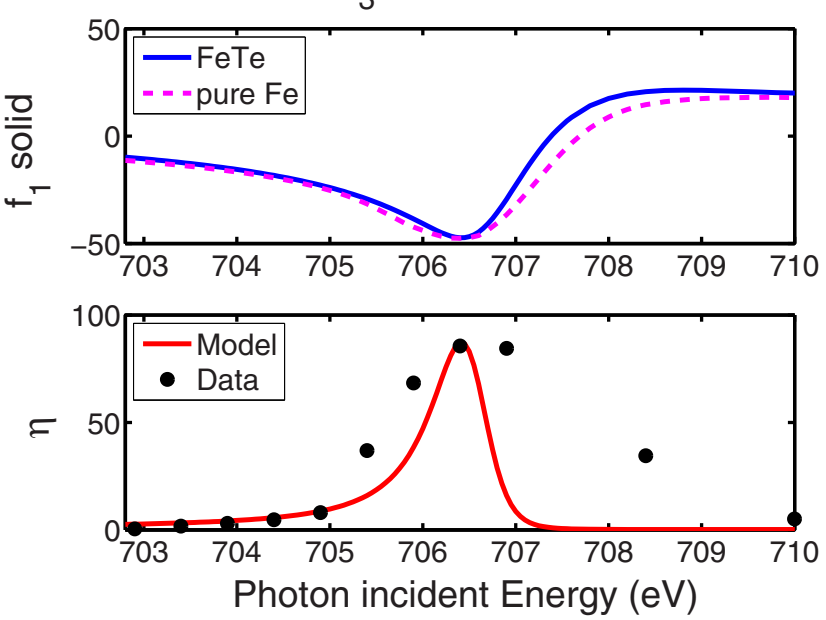

FIG. 2. (Color online) $\mathrm{Fe} L_{3}$ edge of $\mathrm{Fe}_{1.087} \mathrm{Te}$ : In the top frame the solid curve represents the model for $f_{1}$ as a function of the incident energy. The dashed curve represents $f_{1}$ data from total electron yield experiments in pure iron [36]. The label " $f_{1}$ solid" emphasizes that the parameter $f_{1}$ for the solid is different from the one calculated for free atoms. In the bottom frame the black circles and the solid curve are inelastic peak height and the model with $f_{1}$ from FDMNES, respectively, plotted against incident energy. between the two curves is quite impressive. Therefore, the behavior of $f_{1}$ is very similar in $\mathrm{Fe}_{1.087} \mathrm{Te}$ and in pure iron. This result confirms that the $\mathrm{x}$-ray absorption for materials related to iron pnictides is qualitatively similar to Fe metal [37] but also very different from $\mathrm{Fe}$ ions in the $\mathrm{Li}_{x} \mathrm{FePO}_{4}$ compound for the Li-battery cathodes [38], where multiplet calculations must be included [39]. In the bottom frame of Fig. 2 the inelastic peak height is plotted against incident energy together with the theoretical enhancement $\eta$. We have used the value $r_{s}=1.60$ a.u., which is consistent with the plasmon energy losses associated with the Fe $1 s$ core level [40]. The corresponding plasma energy of the Fe $3 d$ shell is $23.3 \mathrm{eV}$. The overall agreement between the experimental data and $\eta$ is good below the threshold energy of $705 \mathrm{eV}$, and it remains reasonable after this threshold despite the fact that the present theory does not include the resonant x-ray emission described by $\mathrm{KH}$. The enhancement factor $\eta$ is very large, and it approaches a value as large as 87 .

In the case of $\mathrm{TiSe}_{2}$, the choice of the parameter $r_{s}=3.0$ yields a screening length $\lambda=\sqrt{1.56 r_{s}}=2.16$ a.u. within the Thomas Fermi model [41], which is consistent with the measured plasma frequency $[42,43]$. This larger $r_{s}$ value gives a much smaller enhancement $\eta$ compared to the FeTe case, which reaches a factor of 14 at most. The FDMNES calculation of $f_{1}$ yields amplitudes of about -77 around an energy of $458 \mathrm{eV}$. In the present case, well-defined particle-hole features given by $S(\mathbf{q}, \omega)$ dominate the fluorescent contributions, in agreement with the findings by Monney et al. [21]. As mentioned above, the $L_{2}$ and $L_{3}$ edges of Ti almost overlap. Therefore, one must perform a calculation for both edges at the same time in order to have an accurate description of the amplitude $f_{1}$. The TDDFT must also be applied in order to describe many-electron effects which modify the ratio of the $L_{2}$ and $L_{3}$ contributions. However, as shown in the bottom of Fig. 3, the agreement with the experiment is still not perfect, despite the model's ability to capture the main amplitude in the RIXS signal.

To illustrate our approach, we calculate the dynamical structure factor $S(\mathbf{q}, \omega)$ for four bands near the Fermi level, as
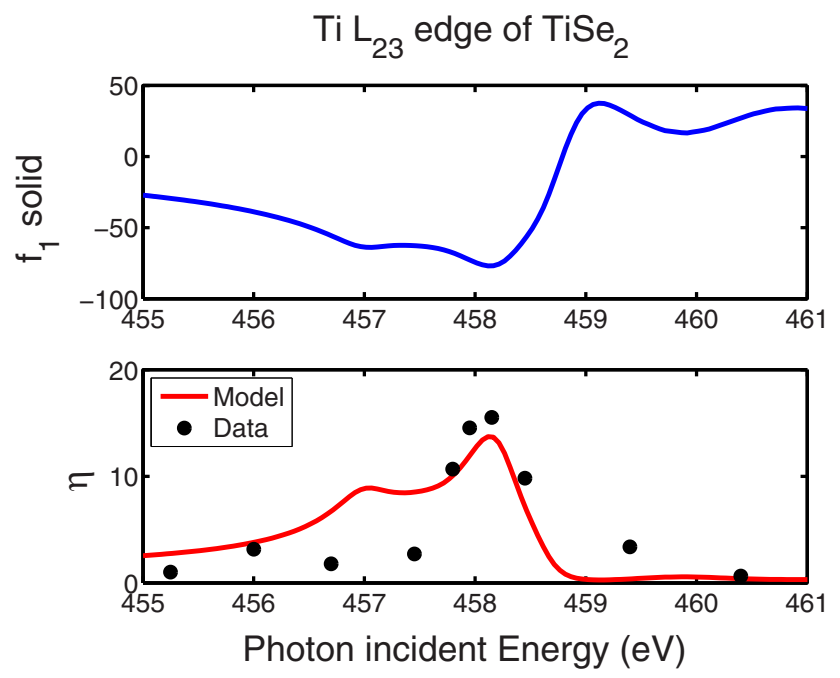

FIG. 3. (Color online) Same as Fig. 2 but for the Ti $L_{23}$ edge of $\mathrm{TiSe}_{2}$ (for an integrated energy-loss region between 0.1 and $0.6 \mathrm{eV}$ ). 
(a)

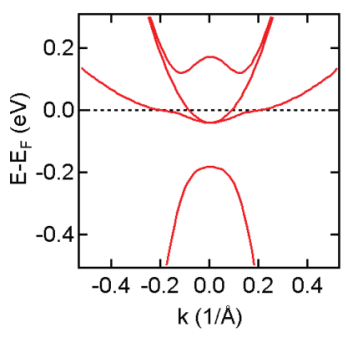

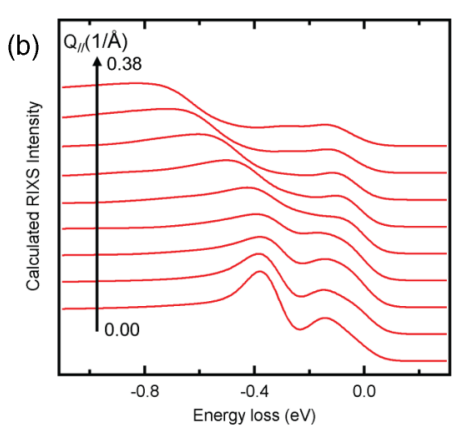

(c)

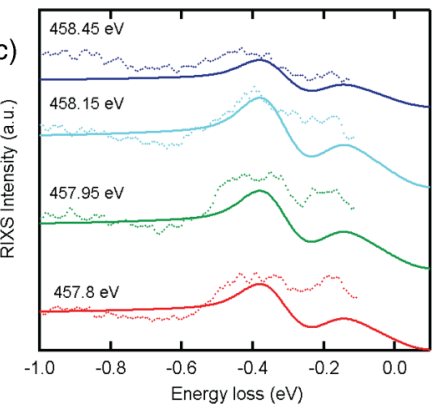

FIG. 4. (Color online) Model for the RIXS in $\mathrm{TiSe}_{2}$ : (a) Band structure given in Ref. [21]. (b) Dispersion of $S(\mathbf{q}, \omega)$. (c) Calculated RIXS spectrum at $q_{\|}=0$ (solid lines), together with experimental RIXS spectra (dotted lines) of $\mathrm{TiSe}_{2}$ for selected incident photon energies, indicated on the left (at $16 \mathrm{~K}$ and for $q_{\|} \sim 0$ ), from Ref. [21] (spectra plotted up to the elastic line).

performed previously [21] to understand electron-hole excitation dispersions in the charge-density-wave phase of $\mathrm{TiSe}_{2}$. These four bands are depicted in Fig. 4(a) and correspond to a model band structure of the charge-density wave in $\mathrm{TiSe}_{2}$, inspired by angle-resolved photoemission spectroscopy experiments. Transitions between the lowest occupied band and the highest unoccupied band give rise to the dispersive peak at high energy losses in RIXS, while transitions between the partially occupied bands and the highest unoccupied one give rise to the dispersive peak at low energy losses. The corresponding spectral functions have been broadened using a Gaussian function to account for the experimental resolution. Figure 4(b) shows the resulting RIXS spectra as a function of the transferred momentum of light projected on the surface plane of the sample, $q_{\|}$. These spectra are given by an energyloss- and momentum-resolved convolution of the occupied and unoccupied band structure, which approximates Eq. (2). Reference [21] shows that such a convolution compares very well with the measured $q$-dependent RIXS spectra for an incident photon energy maximizing the cross section. According to Eq. (1), the cross section can be obtained using the enhancement term $\eta$ given by Eq. (11). Figure 4(c) shows selected calculated RIXS spectra (solid lines) obtained with the dynamical structure factor function at $q_{\|}=0$ and multiplied by the enhancement factor $\eta$ for $\mathrm{TiSe}_{2}$. For comparison, we show in the same panel the corresponding experimental RIXS spectra (dotted lines) of $\mathrm{TiSe}_{2}$ at different incident photon energies (see Ref. [21] for more details). Thus, this example illustrates the present RIXS model for particle-hole excitations in metallic systems. This scheme factorizes the RIXS signal in an incident-energy-dependent part describing the resonance behavior multiplied by an energy-loss- and momentum-dependent term for the excitation dispersion.

\section{CONCLUSIONS}

We propose, by using a renormalization-group approach, to simplify the description of RIXS for the particle-hole excitations in metallic systems. For this purpose, we connect $S(\mathbf{q}, \omega)$ to an effective scattering cross section where the amplification factor $\eta$ is directly related to the atomic scattering parameter $f_{1}(\omega)$, which is strongly modulated across resonant absorption edges. For metallic $\mathrm{Fe}$ and FeTe, the TDDFT calculation of $f_{1}(\omega)$ works very well, while for $\mathrm{TiSe}_{2}$ one can expect some discrepancy between theory and experiment since these calculations usually can reproduce rather well the absorption cross section (related to $f_{1}$ ) for metals but not the absorption in ionic or correlated systems. Nevertheless, we find that the model is still able to capture the overall amplitude of the RIXS signal in $\mathrm{TiSe}_{2}$. The reason for the discrepancies between model and experiment above the absorption thresholds is explained by the fact that the present model does not include the momentum independent resonant emission described by KH. An important question is, what can we learn from the present approach? The band structure calculations for $\mathrm{TiSe}_{2}$ shown by Monney et al. [21] already reproduced many interesting features of the RIXS spectral shape, including the dispersion embedded in $S(\mathbf{q}, \omega)$. However, the missing part of this previous work was the amplitude of the RIXS process involving both energy and momentum conservation, which can now be brought in by our present model. Thus, the most interesting aspect of the present model is a method to extract $S(\mathbf{q}, \omega)$ in metallic systems in future q-dependent RIXS experiments.

\section{ACKNOWLEDGMENTS}

We thank S. Macke for sending us $f_{1}$ data for iron. B.B. is supported by the U.S. Department of Energy (USDOE) Contract No. DE-FG0207ER46352. C.M. and T.S. acknowledge support from the Swiss National Science Foundation (SNSF) and its National Centre of Competence in Research MaNEP. C.M. acknowledges support from the Swiss National Science Foundation (under Grant No. PA00P2_142054) and from the Alexander von Humboldt Foundation. We benefited from computer time from Northeastern University's Advanced Scientific Computation Center (ASCC) and USDOE's NERSC supercomputing center.
[1] E. Isaacs and P. M. Platzman, Phys. Today 49(2), 40 (1996).
[2] P. M. Platzman and N. Tzoar, J. Appl. Phys. 57, 3623 (1985); Phys. Rev. B 2, 3556 (1970). 
[3] W. Schülke, Electron Dynamics by Inelastic X-Ray Scattering (Oxford University Press, Oxford, 2007).

[4] K. Hämäläinen and S. Manninen, J. Phys. Condens. Matter 13, 7539 (2001).

[5] A. Kotani and S. Shin, Rev. Mod. Phys. 73, 203 (2001).

[6] P. Glatzel and U. Bergmann, Coord. Chem. Rev. 249, 65 (2005).

[7] J.-P. Rueff and A. Shukla, Rev. Mod. Phys. 82, 847 (2010).

[8] L. J. P. Ament, M. van Veenendaal, T. P. Devereaux, J. P. Hill, and J. van den Brink, Rev. Mod. Phys. 83, 705 (2011).

[9] J. Laverock, A. R. H. Preston, D. Newby, Jr., K. E. Smith, and S. B. Dugdale, Phys. Rev. B 84, 235111 (2011).

[10] K. Ishii, T. Tohyama, and J. Mizuki, J. Phys. Soc. Jpn. 82, 021015 (2013).

[11] H. Agren and F. Gel'mukhanov, J. Electron Spectrosc. Relat. Phenom. 110-111, 153 (2000).

[12] Y.-P. Sun, F. Hennies, A. Pietzsch, B. Kennedy, T. Schmitt, V. N. Strocov, J. Andersson, M. Berglund, J.-E. Rubensson, K. Aidas, F. Gel'mukhanov, M. Odelius, and A. Föhlisch, Phys. Rev. B 84, 132202 (2011).

[13] J. N. Hancock, G. Chabot-Couture, Y. Li, G. A. Petrakovskiui, K. Ishii, I. Jarrige, J. Mizuki, T. P. Devereaux, and M. Greven, Phys. Rev. B 80, 092509 (2009).

[14] L. Zhang, N. Schwertfager, T. Cheiwchanchamnangij, X. Lin, P.-A. Glans-Suzuki, L. F. J. Piper, S. Limpijumnong, Y. Luo, J. F. Zhu, W. R. L. Lambrecht, and J.-H. Guo, Phys. Rev. B 86, 245430 (2012).

[15] J. Lacki, H. Ruegg, and V. L. Telegdi, Stud. Hist. Philos. Mod. Phys. 30, 457 (1999).

[16] A. Thompson et al., X-ray Data Booklet (Center for X-ray Optics and Advanced Light Source, Lawrence Berkeley National Laboratory, Berkeley, CA, 2009).

[17] W. Van Heringen, Physica (Amsterdam) 26, 306 (1960).

[18] B. Barbiellini and P. Nicolini, Phys. Rev. A 84, 022509 (2011).

[19] M. W. Haverkort, Phys. Rev. Lett. 105, 167404 (2010).

[20] J. N. Hancock, R. Viennois, D. van der Marel, H. M. Ronnow, M. Guarise, P.-H. Lin, M. Grioni, M. Moretti Sala, G. Ghiringhelli, V. N. Strocov, J. Schlappa, and T. Schmitt, Phys. Rev. B 82, 020513(R) (2010).

[21] C. Monney, K. J. Zhou, H. Cercellier, Z. Vydrova, M. G. Garnier, G. Monney, V. N. Strocov, H. Berger, H. Beck, T. Schmitt, and P. Aebi, Phys. Rev. Lett. 109, 047401 (2012).

[22] M. Magnuson, J.-E. Rubensson, A. Föhlisch, N. Wassdahl, A. Nilsson, and N. Martensson, Phys. Rev. B 68, 045119 (2003).
[23] P. Eisenberger, P. M. Platzman, and H. Winnick, Phys. Rev. Lett. 36, 623 (1976).

[24] J. P. Reed, B. Uchoa, Young Il Joe, Yu Gan, D. Casa, E. Fradkin, and P. Abbamonte, Science 330, 805 (2010).

[25] Y. J. Wang, B. Barbiellini, H. Lin, T. Das, S. Basak, P. E. Mijnarends, S. Kaprzyk, R. S. Markiewicz, and A. Bansil, Phys. Rev. B 85, 224529 (2012).

[26] M. P. Prange, J. J. Rehr, G. Rivas, J. J. Kas, and John W. Lawson, Phys. Rev. B 80, 155110 (2009).

[27] M. Sacchi, C. F. Hague, E. M. Gullikson, and J. H. Underwood, Phys. Rev. B 57, 108 (1998).

[28] K. H. Stone, S. M. Valvidares, and J. B. Kortright, Phys. Rev. B 86, 024102 (2012).

[29] O. Bunau and Y. Joly, J. Phys. Condens. Matter 21, 345501 (2009).

[30] O. Bunau and Y. Joly, Phys. Rev. B 85, 155121 (2012).

[31] V. N. Strocov, T. Schmitt, U. Flechsig, T. Schmidt, A. Imhof, Q. Chen, J. Raabe, R. Betemps, D. Zimoch, J. Krempasky, X. Wang, M. Grioni, A. Piazzalunga, and L. Patthey, J. Synchrotron Radiat. 17, 631 (2010).

[32] G. Ghiringhelli, A. Piazzalunga, C. Dallera, G. Trezzi, L. Braicovich, T. Schmitt, V. N. Strocov, R. Betemps, L. Patthey, X. Wang, and M. Grioni, Rev. Sci. Instrum. 77, 113108 (2006).

[33] A. Subedi, L. Zhang, D. J. Singh, and M. H. Du, Phys. Rev. B 78, 134514 (2008).

[34] Y. J. Wang, H. Lin, B. Barbiellini, P. E. Mijnarends, S. Kaprzyk, R. S. Markiewicz, and A. Bansil, Phys. Rev. B 81, 092501 (2010).

[35] Y. J. Wang, H. Lin, B. Barbiellini, P. E. Mijnarends, S. Kaprzyk, W. Al-Sawai, R. S. Markiewicz, and A. Bansil, J. Supercond. Novel Magn. 22, 569 (2009).

[36] S. Macke (private communication).

[37] W. L. Yang et al., Phys. Rev. B 80, 014508 (2009).

[38] A. Hunt, W.-Y. Ching, Y.-M. Chiang, and A. Moewes, Phys. Rev. B 73, 205120 (2006).

[39] X. Liu et al., J. Am. Chem. Soc. 134, 13708 (2012).

[40] N. Moslemzadeh, G. Beamson, P. Tsakiropoulos, and J. F. Watts, Surf. Sci. 600, 265 (2006).

[41] B. Barbiellini-Amidei, Phys. Lett. A 134, 328 (1989).

[42] G. Li, W. Z. Hu, D. Qian, D. Hsieh, M. Z. Hasan, E. Morosan, R. J. Cava, and N. L. Wang, Phys. Rev. Lett. 99, 027404 (2007).

[43] C. Monney, G. Monney, P. Aebi, and H. Beck, New J. Phys. 14, 075026 (2012). 